NOTE

\title{
Exponential and linear estimations of grazing on bacteria: effects of changes in the proportion of marked cells
}

\author{
Jordi Salat, Cèlia Marrasé \\ Institut de Ciències del Mar, CSIC, Passeig Nacional s/n, E-08039 Barcelona, Spain
}

\begin{abstract}
We develop a method to calculate grazing on bacteria determined by the disappearance of marked cells or particles. The method uses an exponential model based on population dynamics. We also describe 2 linear approaches: one is a model currently in use that takes into account only the initial proportion of marked cells; the other also includes their final proportion. An experimental data set is used to compare the 3 models. The first linear model tends to underestimate grazing due to lack of information concerning the final proportion of marked cells. The second linear model, which does take into account the final abundances of marked and natural cells, results in a good approximation to the exponential. We conclude that consideration of the final proportion of marked to natural cells is crucial to correctly estimate bacterial grazing.
\end{abstract}

KEY WORDS: Bacterivory - Mathematical methods - FLB

The contribution of bacteria to the total plankton biomass in aquatic ecosystems is frequently substantial (Ferguson \& Rublee 1976, Watson et al. 1977, Holligan et al. 1984, Fuhrman et al. 1989). Thus, in modeling energy flow in these ecosystems it is necessary to quantify bacterial mortality from predation.

Several methods to quantify the rate of total consumption of bacteria have been described (e.g. Landry et al. 1984, Wright \& Coffin 1984, Sherr et al. 1986). Lately, methods which rely on the disappearance of marked cells over an extended incubation (24 to $48 \mathrm{~h}$ ) have frequently been used (Pace et al. 1990, Marrasé et al. 1992, Vaqué et al. 1992). These methods are based on adding dead marked cells to natural unfiltered water volumes, at concentrations of 10 to $40 \%$ of the abundance of natural bacteria. Water samples are then incubated over 24 to $48 \mathrm{~h}$ and subsamples are taken at the beginning and at the end of the incubation period.

Conceptually, during the incubation period, the abundance of bacteria and marked cells follow expo- nential models. The percentage of marked cells changes over this period because the natural bacteria reproduce. Nevertheless, this aspect was ignored in recently published papers (Pace et al. 1990, Vaqué et al. 1992). Simply the initial percentage of marked cells was used to calculate the grazing (Vaqué pers. comm.).

Here, we compare 3 different models to calculate grazing activity: (1) a linear model taking into account the initial concentrations of marked and natural cells and the final concentration of marked cells; (2) a linear model taking into account the initial and final concentration of both marked and natural cells; (3) an exponential model based on population dynamics. We apply these 3 models to an experimental data set to illustrate the different grazing calculated with each model. Finally we demonstrate analytically isee appendix) that the second model is a fairly good approximation to the third.

Description of models. Model 1: In this model it is assumed that the percentage of marked cells with respect to the natural bacteria does not change over the incubation period. This assumption implies that reproduction of natural bacteria is negligible. Authors who use this model calculate grazing rates as follows:

$$
G=\left(F_{0}-F_{\Upsilon}\right) \frac{N_{0}}{F_{0}}
$$

where $G=$ number of bacteria grazed during the period $T_{;} F_{0}$ and $F_{T}=$ the initial and final abundances of marked cells; and $N_{0}=$ initial abundance of natural bacteria. Thus, grazing is calculated by multiplying the decrease in marked cells by the initial percentage of marked to natural cells.

Model 2: Here it is assumed that the mean numbers of natural $(\bar{N})$ and marked $(\bar{F})$ cells over a period $T$ coincide with the arithmetic mean of the values at the 
beginning and at the end of the incubation (linear evolution). That is,

$$
\bar{N}=\frac{N_{0}+N_{T}}{2} \text { and } \bar{F}=\frac{F_{0}+F_{T}}{2} .
$$

The proportion of marked bacteria removed by grazing during the period $T$ will be

$$
q=\frac{F_{0}-F_{T}}{\bar{F}} .
$$

Now, if we assume that the natural bacteria and marked cells are removed at the same rate, the number of natural bacteria grazed during the period $T$ can be calculated as follows:

$$
G=q \bar{N}=\left(F_{0}-F_{T}\right)\left(\frac{N_{0}+N_{T}}{F_{0}+F_{T}}\right) .
$$

Model 3: This is based on the population dynamics of the bacterial assemblage (Marrasé et al. 1992). Considering the dynamics of living bacteria, the net growth of the population at time $t$ can be expressed as

$$
\frac{\mathrm{d} N}{\mathrm{~d} t}=\frac{\mathrm{d} C}{\mathrm{~d} t}-\frac{\mathrm{d} P}{\mathrm{~d} t}
$$

where $N=$ total number of cells at time $t_{i} C=$ number of new cells produced; and $P=$ number of predated cells.

If we assume that growth and predation are proportional to the total number of cells, we can express

$$
\frac{\mathrm{d} C}{\mathrm{~d} t}=k N \text { and } \frac{\mathrm{d} P}{\mathrm{~d} t}=g N .
$$

where $k$ and $g$ are constants. Then

$$
\frac{\mathrm{d} N}{\mathrm{~d} t}=k N-g N=a N,
$$

where $a=k-g$ is also constant. We can call $a$ the net instantaneous growth rate, and $k$ and $g$ the instantaneous gross growth and grazing rates respectively. Notice that if $a>0$ the population grows, and if $a<0$ the population is driven to collapse. If $a=0$, the population is stable; growth and predation are balanced.

Integrating Eq. (7), for $a \neq 0$,

$$
\frac{\mathrm{d} N}{N}=a \mathrm{~d} t, \text { then } N=N_{0} \mathrm{e}^{a t},
$$

and, substituting Eq. (8) in Eq. (6a, 6b), we have

$$
\frac{\mathrm{d} C}{\mathrm{~d} t}=k N_{0} \mathrm{e}^{a t} \text { and } \frac{\mathrm{d} P}{\mathrm{~d} t}=g N_{0} \mathrm{e}^{a t} .
$$

The net growth of the population over a period $T$ can be obtained from Eq. (8) as

$$
\Delta N=N_{T}-N_{0}=N_{0} \mathrm{e}^{a t}-N_{0}=N_{0}\left(\mathrm{e}^{a t}-1\right) .
$$

The gross growth $\Delta C$ and predation $\Delta P$ during this period can be obtained by integrating Eq. $(9 a, 9 b)$ as follows:

$$
\Delta C=\int_{0}^{T} \mathrm{~d} C=\int_{0}^{T} k N_{0} \mathrm{e}^{a t} \mathrm{dt}=\frac{k N_{0}}{a}\left(\mathrm{e}^{a T}-1\right)=\frac{k}{a} \Delta N .
$$

Similarly,

$$
\Delta P=\frac{g}{a} \Delta N
$$

Consequently,

$$
\Delta C-\Delta P=\left(\frac{k}{a}-\frac{g}{a}\right) \Delta N=\Delta N
$$

which is an obvious result.

Note that if $a=0, g=k$ and $\Delta C=\Delta P=g N_{0} T$.

Now let us go back to consider the dynamics of natural and marked bacteria. Natural bacteria will both grow and suffer predation while marked cells will only be predated. If we assume that predators are not selective (the same assumption as in Models 1 and 2), then predation rates are the same for both assemblages.

In this case, during a period of time $T$, according to Eq. (8),

$$
N_{T}=N_{0} \mathrm{e}^{a T} \text { and } F_{T}=F_{0} \mathrm{e}^{-g T},
$$

where $N$ refers to the natural bacteria and $F$ to marked cells (as before). Observe that the net growth of marked cells is $0-g=-g$.

From a practical point of view, the method of calculating grazing according to this model will be as follows. First, we determine, from Eq. (14), the net instantaneous growth and grazing rates ( $a$ and $g$ respectively):

$$
a=\frac{1}{T} \ln \left(\frac{N_{T}}{N_{0}}\right) \text { and } g=-\frac{1}{T} \ln \left(\frac{F_{T}}{F_{0}}\right)
$$

where $N_{0}, N_{T}, F_{0}$ and $F_{T}$ are obtained experimentally; and, second, we use them to calculate the number of natural bacteria predated during this period, according to Eqs. (12) and (10):

For $a \neq 0$,

$$
G=\Delta P=\frac{g}{a} \Delta N=\frac{g}{a}\left(N_{T}-N_{0}\right) .
$$

and, for $a=0$,

$$
G=\Delta P=g N_{0} T .
$$

Formal similarities among the three models: Observe that one similarity among the 3 models of $G$ is that each contains $\left(F_{0}-F_{T}\right)$ multiplied by a factor (Table 1). In the first model, this factor is the inverse of the initial proportion of marked particles; in the second and the third models the inverse proportion is obtained using the means (linear and exponential respectively) 
Table 1. Formal similarities among the 3 models. $G$ : bacteria grazed during a period $T_{;} N_{0}$ and $N_{T}$ : natural bacteria abundance at time 0 and time $T$ respectively; $F_{0}$ and $F_{T}$ : marked particle abundance at time 0 and time $T$ respectively

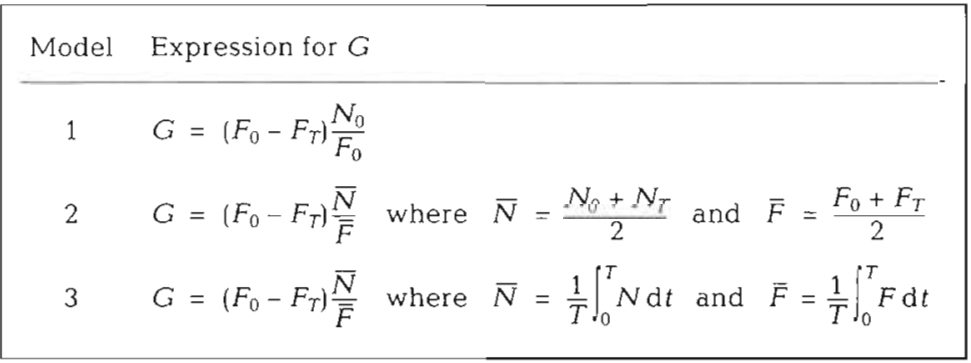

over the period (Table 1). The first 2 expressions of $G$ in Table 1 are given explicitly in the sections above and the third can be easily shown as follows:

$\bar{N}=\frac{1}{T} \int_{0}^{T} N \mathrm{~d} t=\frac{1}{T} \int_{0}^{T} N_{0} \mathrm{e}^{a t} \mathrm{~d} t=\frac{N_{0}}{a T}\left(\mathrm{e}^{a T}-1\right)=\frac{\Delta N}{a T}$.

then, from Eq. (12),

$$
G=\Delta P=\frac{g}{a} \Delta N=g \bar{N} T .
$$

Similarly,

$\bar{F}=\frac{1}{T} \int_{0}^{T} F \mathrm{~d} t=\frac{1}{T} \int_{0}^{T} F_{0} \mathrm{e}^{-g t} \mathrm{~d} t=\frac{F_{0}}{g T}\left(1-\mathrm{e}^{-g T}\right)=\frac{1}{g T}\left(F_{0}-F_{T}\right)$,

then,

$$
T=\frac{1}{g \bar{F}}\left(F_{0}-F_{T}\right)
$$

substituting Eq. (21) in Eq. (19), we obtain:

$$
G=\frac{g \bar{N}}{g \bar{F}}\left(F_{0}-F_{T}\right)
$$

Comparison of models. We have used data from Marrasé et al. (1992) to examine the differences among models. In that paper, the seasonal and daily changes in bacterivory in a coastal plankton community (Vineyard Sound, Massachusetts, USA) were studied, and authors calculated community grazing rates from the disappearance of Fluorescently Labeled Bacteria (FLB). This data set, which included 69 cases, is the only one we are aware of that has natural and labeled bacterial abundance at both the beginning and the end of the incubation period (in this case $48 \mathrm{~h}$ ). For Model 3, the range of values of the instantaneous grazing and gross growth rates varied between 0.02 and $0.6 \mathrm{~d}^{-1}$ and between 0.03 and $0.8 \mathrm{~d}^{-1}$ respectively.

We calculated total community grazing rates $(G)$ from this data set using the 3 models and compared the results obtained from each linear model (Models 1 and 2) against the exponential model (Model 3) with a regression analysis. In both analyses the amount of variance explained by the regression was high $(\mathrm{n}=69$, Model 3 vs 1 : $r^{2}=0.947$; Model 3 vs $2: r^{2}=0.998$ ), but the slopes were different. As was expected from the theoretical comparison described in the appendix, results from Models 2 and 3 showed good agreement (Fig. 1A, slope = 1.023), while results from Model 1 were systematically lower than those calculated by Model 3 (Fig. 1B, slope = 1.536).

The discrepancies between Model 1 and the other 2 models arose from the fact that Model 1 does not consider the final abundance of the natural bacterial assemblage. Consequently, if gross growth rate, $k$, is close to 0 (i.e. $N_{0} / F_{0} \approx N_{T} / F_{T}$ ) the behaviour of the 3 models is approximately the same. In particular, when $k=0$ $\left(N_{0} / F_{0}=N_{T} / F_{T}\right)$, Eqs. (4) and (1) coincide.

To show how the discrepancies varied for different grazing and gross growth rates, we examined, as an example, the variation of the quotient between results from Model 1 and Model 3 for the 2 extreme $g$ values $\left(0.02\right.$ and $\left.0.6 \mathrm{~d}^{-1}\right)$, when varying the $k$ values from 0 to
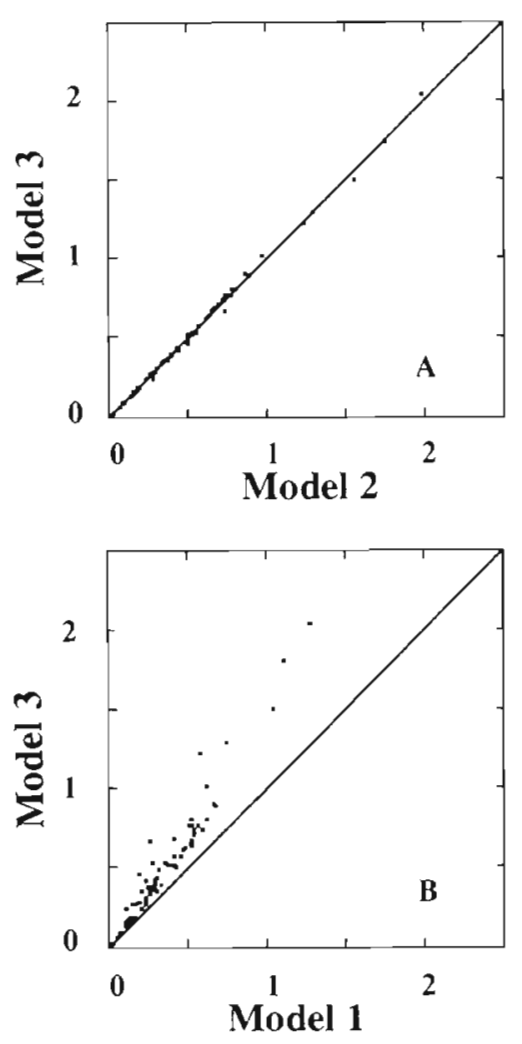

Fig. 1. Comparison of 3 estimates of total community grazing rates ( $G$; bacteria $\times 10^{6} \mathrm{ml}^{-1} \mathrm{~d}^{-1}$ ). (A) Model 2 vs 3; (B) Model 1 vs 3 . Note that the line is just $y=x$ and not the regression line 


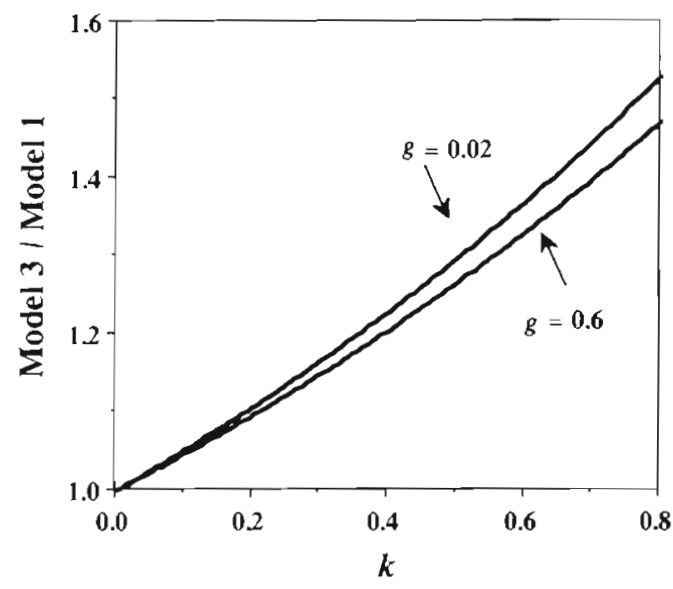

Fig. 2. Variation of the quotient between results from Model 1 and Model 3 for the 2 extreme $g$ values $\left(0.02\right.$ and $\left.0.6 \mathrm{~d}^{-1}\right)$ when $k$ varies from 0 to $0.8 \mathrm{~d}^{-1}$

0.8 (Fig. 2). These discrepancies are almost negligible when varying $g$, but, as previously mentioned, the differences between models magnify as $k$ increases. Note that for a likely value of $k=0.5$, the underestimation of grazing from Model 1 already reaches $20 \%$ per day.

Discussion. Several experimental protocols used to evaluate grazing on bacteria, such as the disappearance of labeled cells, require long-term incubations (24 to $48 \mathrm{~h}$ ). Using these protocols, any analytical model to quantify the bacteria grazed should take into account both the grazing process and the growth of natural bacteria. We compared 3 different approaches to calculate grazing on bacteria. The linear approach of Model 1 (currently in use) does not take into account the growth of natural bacteria, while both Model 2 (linear) and Model 3 (exponential) approaches do take into account changes in natural bacteria abundances.

From a conceptual point of view, we consider that the best approximation is obtained using an exponential model (Model 3). In fact, the main reason for including Model 2 here is to illustrate that the kind of approach chosen (linear vs exponential) to estimate grazing is not as relevant as is the lack of information about final bacterial abundance (Model 1). This is seen from the comparison of models (Model 1 vs 3 and 2 vs 3 ; Fig. 1).

We have therefore proved that Model 2 is a good linear approximation to the exponential, which could be used alternatively with certain limitations (see Eq. A13 in the appendix). However, we recommend the exponential model because it also gives information on both grazing and growth processes through instantaneous rates ( $g$ and $k$ respectively).

We have also proved that when the final abundance of natural bacteria is not considered (Model 1), the number of bacteria grazed will always be underestimated (or overestimated when gross growth rate, $k_{1}$ is negative). In any case, differences between Model 1 and the others will be greater with greater difference of $k$ from zero. As many studies show fairly rapid growth of bacteria, we discourage the use of Model 1. The underestimation of grazing in these cases becomes large (at least $20 \%$ per day for $k \geq 0.5$; Fig. 2).

In conclusion, for accurate measurements of grazing, the final counts of natural bacteria should be determined, especially in systems where growth is expected to be relatively high (eutrophic situations) or negative (situations in which there are other important mortality sources, e.g. viruses).

Acknowledgements. We thank D. Vaqué, C. Pedrós-Alió, M. MacLean, W. Norbis, J. Martínez de Albéniz and 3 anonymous referees for their helpful comments. This work was supported by CICYT grant MAR91-0359

\section{LITERATURE CITED}

Ferguson, R. L., Rublee, P. (1976). Contribution of bacteria to standing crop of coastal plankton. Limnol. Oceanogr. 21: $141-145$

Fuhrman, J. A. Sleeter, T. D., Carlson, C. A., Practor, L. M. (1989). Dominance of bacteria biomass in the Sargasso Sea and its ecological implications. Mar. Ecol. Prog. Ser. 57: $207-217$

Holligan, P. M., Harris, R. P., Newell, R. C., Harbour, D. S., Head, R. N., Linley, E. A. S., Lucas, M. I., Tranter, P. R. G. Weekly, C. M. (1984). Vertical distribution and partitioning of organic carbon in mixed, frontal and stratified waters of the English Channel. Mar. Ecol. Prog. Ser. 14: $111-127$

Landry, M. R., Hass, L. W., Fagerness, V. L. (1984). Dynamics of microbial plankton communities: experiments in Kaneohe Bay, Hawaii. Mar. Ecol. Prog. Ser. 16: 127-133

Marrasé, C., Lim, E. L., Caron, D. A. (1992). Seasonal and daily changes in bacterivory in a coastal plankton community. Mar. Ecol. Prog. Ser. 82: 281-289

Pace, M. L., McManus, G. B., Findlay, S. E. G. (1990). Planktonic community structure determines the fate of bacterial production in a temperate lake. Limnol Oceanogr. 35 . $795-808$

Sherr, B. F., Sherr, E. B., Andrew, T. L., Fallon, R. D., Newell, S. Y (1986). Trophic interactions between heterotrophic Protozoa and bacterioplankton in estuarine water analyzed with selective metabolic inhibitors. Mar. Ecol. Prog. Ser. 32: 169-179

Vaqué, D., Pace, M. L., Findlay, S. E. G., Lints, D. (1992). Fate of bacterial production in a heterotrophic ecosystem: grazing by protists and metazoans in the Hudson Estuary. Mar. Ecol. Prog. Ser. 89: 155-163

Watson, S. W., Novitsky, T. J., Quinby, H. L., Valois, F. W. (1977). Determination of bacterial number and biomass in the marine environment. Appl. environ. Microbiol. 33: $940-946$

Wright, R. T., Coffin, R. B. (1984). Measuring microzooplankton grazing on planktonic marine bacteria by its impact on bacterial production. Microb. Ecol. 10: 137-149 


\section{APPENDIX}

\section{Comparison between Model 2 and Model 3}

In this section we compare Models 2 and 3 analytically. First, we find that instantaneous rates, $a$ and $g$, can be approximated by a function in a neighbourhood of 0 through Taylor development. This function was chosen so that the resulting approximated values $a^{\prime}$ and $g^{\prime}$ could be obtained linearly from $N_{0}, N_{r}$, and $F_{0}, F_{r}$.

Next, we show that the expression for grazing $(G)$ in Model 2 can be converted formally to the same expression from Model 3 using these approximate values. Finally, we determine the intervals of $g$ and $a$ values that approximate the function $G$ with an error less than $10 \%$.

Let us consider $y(x)$ to be the following function:

$$
y=2 \frac{\mathrm{e}^{x}-1}{\mathrm{e}^{x}+1}
$$

The derivative of $y(x)$ is

$$
y^{\prime}=\frac{\mathrm{d} y}{\mathrm{~d} x}=\frac{4 \mathrm{e}^{x}}{\left(\mathrm{e}^{x}+1\right)^{2}},
$$

and the second derivative is

$$
y^{\prime \prime}=\frac{\mathrm{d}^{2} y}{\mathrm{~d} x^{2}}=\frac{4 \mathrm{e}^{x}\left(1-\mathrm{e}^{x}\right)}{\left(\mathrm{e}^{x}+1\right)^{3}} .
$$

Developing the function $y(x)$ in a Taylor series, in a neighbourhood of 0 ,

$$
y(x)=y(0)+y^{\prime}(0) x+\frac{y^{\prime \prime}(0)}{2 !} x^{2}+\ldots
$$

and, from Eqs. (A1), (A2) and (A3),

$$
y(0)=0, \quad y^{\prime}(0)=\frac{4}{4}=1, \text { and } y^{\prime \prime}(0)=0,
$$

then,

$$
y(x)=x+\varepsilon(x) .
$$

It can be easily demonstrated that the function satisfies the following properties (Fig. A1):

$$
\begin{aligned}
& \text { (i) } y(0)=0 \\
& \text { (ii) } y(-x)=-y(x) \\
& \text { (iii) }|y(x)|<|x|, x \neq 0 \\
& \text { (iv) }|\varepsilon(x)| \text { is a monotonously increasing function of }|x| \text {. }
\end{aligned}
$$

Now, we calculate that the value of $x$ which gives $|\varepsilon(x)|=0.1$ is $x=1.16$, and, according to (i) to (iv), we can be sure that

$$
|\varepsilon(x)|<0.1, \text { for }|x|<1.16,
$$

so that $y(x)$ is an approximation to $x$ with an error less than $10 \%$ when $|x|<1.16$.

Now define $a^{\prime}$ and $g^{\prime}$ as approximations of $a$ and $g$ respectively, according to Eq. (A1):

$$
a^{\prime} T=2 \frac{\mathrm{e}^{a T}-1}{\mathrm{e}^{a T}+1} \text { and } g^{\prime} T=2 \frac{\mathrm{e}^{g^{T}}-1}{\mathrm{e}^{g T}+1} .
$$

Fig. A1 The graph of the function (a) $y=x$ and (b) its approximation.

$$
y=2 \frac{\mathrm{e}^{x}-1}{\mathrm{e}^{x}+1}
$$

in a neighbourhood of 0

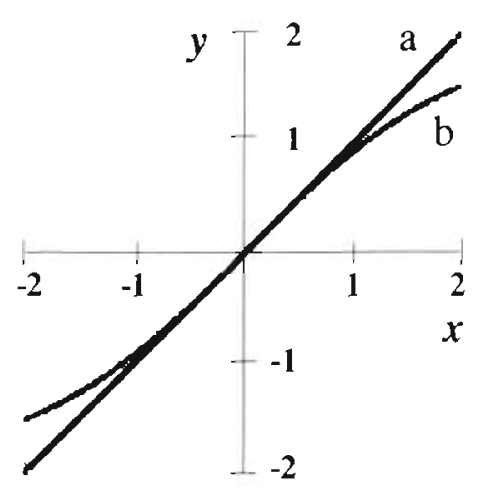

Then,

$$
a^{\prime}=\frac{2}{T} \frac{N_{0}\left(\mathrm{e}^{a T}-1\right)}{N_{0}\left(\mathrm{e}^{a T}+1\right)}=\frac{2}{T} \frac{N_{T}-N_{0}}{N_{T}+N_{0}}
$$

and

$$
-g^{\prime}=\frac{2}{T} \frac{F_{0}\left(\mathrm{e}^{-g T}-1\right)}{F_{0}\left(\mathrm{e}^{-g r}+1\right)}=\frac{2}{T} \frac{F_{r}-F_{0}}{F_{T}+F_{0}}
$$

Substituting these approximations of $a$ and $g$ in Eq. (16), we have

$$
G^{\prime}=\frac{g^{\prime}}{a^{\prime}} \Delta N=\frac{\frac{F_{0}-F_{T}}{F_{0}+F_{T}}}{\frac{N_{T}-N_{0}}{N_{T}+N_{0}}}\left(N_{T}-N_{0}\right)=\left(F_{0}-F_{T}\right) \frac{N_{T}+N_{0}}{F_{0}+F_{T}},
$$

which coincides with Eq. (4), the expression for grazing from Model 2.

Now, we can compare the expressions for grazing $G^{\prime}$ (Eq. A11) from Model 2 and $G$ for Model 3 (Eq. 16):

$$
\frac{G^{\prime}}{G}=\frac{g^{\prime}}{a^{\prime}} \Delta N / \frac{g}{a} \Delta N=\frac{g^{\prime}}{a^{\prime}} \frac{a}{g}
$$

Then, if $|a T|<1.16$ and $g T<1.16$, according to Eq. (A7),

$$
1<\frac{g}{g^{\prime}}<1.1 \text { and } 1<\frac{a}{a^{\prime}}<1.1 \text {. }
$$

therefore,

$$
\frac{1}{1.1}<\frac{G^{\prime}}{G}<1.1
$$

In summary, the linear Model 2 approximates Model 3 with an error of less than $10 \%$ for:

$$
\ln \left(F_{0}\right)-\ln \left(F_{T}\right)<1.16 \text { and }\left|\ln \left(N_{0}\right)-\ln \left(N_{T}\right)\right|<1.16,
$$
or,

$$
\frac{F_{0}}{F_{T}}<3.2 \text { and } 0.3<\frac{N_{T}}{N_{0}}<3.2
$$

